\title{
Liquid Crystals based on carboxylic acids, amines and nitro compounds derived from 1,3,4-thiadiazole.
}

\author{
M. L. Parra ${ }^{a}$, E. Y. Elguetaa ${ }^{a}$ A. I. Sánchez ${ }^{a}$, J. M. Vergara ${ }^{a}$, P. I. Hidalgo ${ }^{a}$, J. A. Ulloa ${ }^{*}$. \\ ${ }^{a}$ Facultad de Ciencias Químicas, Departamento de Química Orgánica, Universidad de Concepción, Casilla \\ 160-C, Concepción, Chile \\ joseulloa@udec.cl
}

Keywords: Liquid Crystals, 1,3,4-thiadiazole, Columnar LC.

\section{INTRODUCTION}

Heterocycles are of great importance in thermotropic liquid crystals. ${ }^{1}$ Mesogens containing 1,3,4thiadiazole have significant lateral dipole moments, wide mesomorphic temperature ranges, chemical stability and altering the molecular shape, leading to different liquid crystal behaviours. ${ }^{2}$ Moreover, the generation of columnar liquid crystals exhibit a molecular organization that allows the transport of energy or charge. In this abstract, we report the synthesis, characterization and mesomorphic properties of novel carboxylic acids (III a-b), amines (II $\mathbf{a}-\mathbf{b}$ and IV $\mathbf{a}-\mathbf{b})$ and nitro compounds (I $\mathbf{a}-\mathbf{b}$ ) derived from 1,3,4-thiadiazole displaying liquid crystal properties. The synthetic route is shown in scheme 1.

\section{RESULTS AND DISCUSSION}
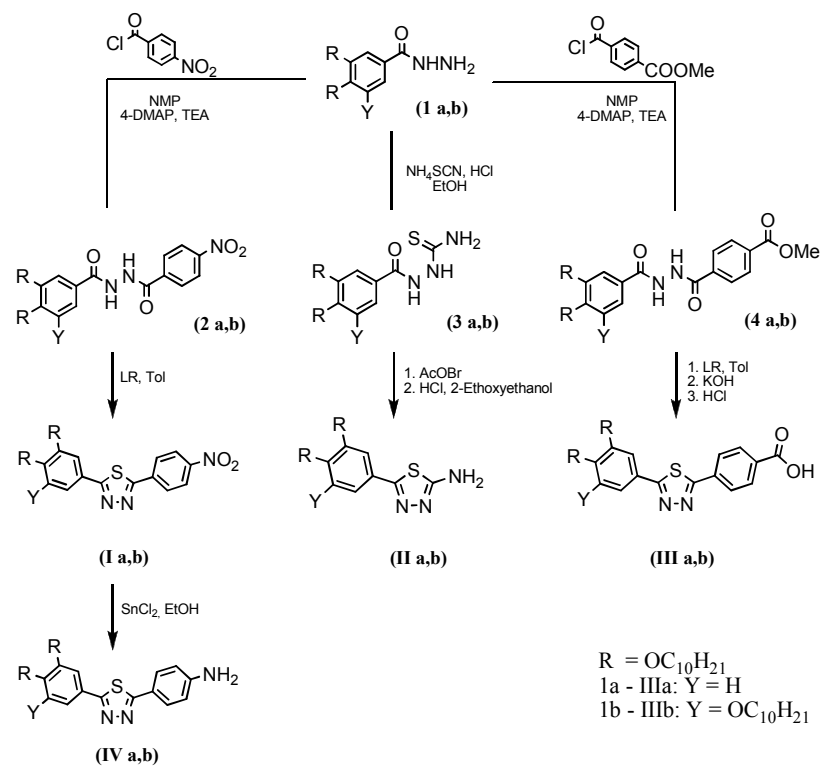

Scheme 1. Synthesis of mesogens I a,b - IV a,b. LR = Lawesson Reagent.

For compounds of the series $(\mathbf{I} \mathbf{a}, \mathbf{b})$ the results are mixed, the compound la do not show liquid crystal properties, Contrary to this compound $\mathbf{l b}$ shows columnar liquid crystal properties. Both compounds of series (II $\mathbf{a}, \mathbf{b})$ and
(III a,b) display mesomorphic properties, in each case a columnar mesophase was observed under optical polarizing microscopy (see figure 1). For compounds of series (III a,b) the columnar mesophase was identified by $x$-ray diffraction as hexagonal columnar phase. These mesomorphic properties of acids (III $\mathbf{a}, \mathbf{b})$ are attributed to intermolecular hydrogen bonding between two molecules of acid which in turn dimerize giving a policatenar mesogen. The series (IV a,b) also show mesomorphic properties, when compared with the analogous (II a,b), we found that additional benzene ring in (IV $\mathbf{a}, \mathbf{b})$ produces higher melting temperatures and lower clearing temperatures (see table 1).
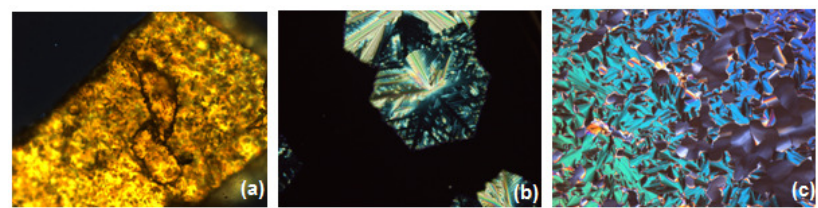

Figure 1: Optical micrographs (Magnification: 40x) of (a) IVb at $40^{\circ} \mathrm{C}$; (b) IIIa at $166^{\circ} \mathrm{C}$; (c) IIlb at $136^{\circ} \mathrm{C}$

Table 1. Transition temperatures $\left[{ }^{\circ} \mathrm{C}\right]$. Col $h=$ hexagonal columnar mesophase, $\mathrm{Col}_{\mathrm{x}}=$ unidentified columnar mesophase, $\mathrm{RT}=$ Room temperature

\begin{tabular}{|c|c|c|}
\hline & a & b \\
\hline I & Cr 174 Iso & Cr 68 Col 72 Iso \\
\hline II & Cr 83 Col 120 Iso & RT Col 164 Iso \\
\hline III & Cr 144 Col 235 Iso & Cr 124 Col 132 Iso \\
\hline IV & Cr 92 Col 103 Iso & Cr 43 Col 59 Iso \\
\hline
\end{tabular}

\section{CONCLUSION}

In conclusion, the 1,3,4-thiadiazole reported here showing good mesomorphic properties. The best results were obtained with molecules capable of generating intermolecular hydrogen bonding.

\section{ACKNOWLEDGEMENTS}

This work was supported by FONDECYT (Grant 1100140), CONICYT (Postgraduate Scholarship) and "Dirección de Investigación" of the University of Concepción.

\section{REFERENCES}

${ }^{1}$ Seed, A., Chem. Soc. Rev., 2007, 36, 2046-2069.

${ }^{2}$ Han, J.; Chang X. Y.; Zhu L. R.; Wang Y. M.; Meng J. B.; Lai S. W. Chui S.S.Y., Liq. Cryst., 2008, 35, 1379-1394. 